\title{
Measurement of the transverse momentum spectrum of the Higgs boson decaying into WW at 8 TeV with the CMS detector
}

\author{
Lorenzo Viliani*t \\ INFN Sezione di Firenze, Università di Firenze, Firenze, Italy \\ Also at CERN, European Organization for Nuclear Research, Geneva, Switzerland \\ E-mail: lorenzo.viliani@cern.ch
}

\begin{abstract}
Differential and integrated fiducial cross sections measured using the Higgs to $\mathrm{W}^{+} \mathrm{W}^{-}$leptonic decays are presented as a function of the Higgs boson production. The measurements are performed using proton-proton collisions at a centre-of-mass energy of $8 \mathrm{TeV}$ collected by the CMS experiment at the LHC, corresponding to an integrated luminosity of $19.4 \mathrm{fb}^{-1}$. The Higgs boson transverse momentum is reconstructed using the lepton pair transverse momentum and missing transverse momentum, which originates from the presence of two neutrinos in the final state. The differential cross section is measured as a function of the Higgs boson transverse momentum in a fiducial phase space defined to match the experimental acceptance in terms of the lepton kinematics and event topology. The measurements are compared to theoretical calculations.
\end{abstract}

Fourth Annual Large Hadron Collider Physics

13-18 June 2016

Lund, Sweden

${ }^{*}$ Speaker.

${ }^{\dagger}$ On behalf of the CMS Collaboration 


\section{Introduction}

Measurements of the production cross section of the Higgs boson times branching fraction of decay in a restricted part of the phase space (fiducial phase space), together with its kinematic properties, represent an important test for possible deviations from the SM predictions. In particular, it has been shown that the Higgs boson transverse momentum $\left(p_{\mathrm{T}}^{\mathrm{H}}\right)$ spectrum can be significantly affected by the presence of physics interactions not predicted by the SM [1]. Moreover, these measurements also allow stringent tests of the theoretical predictions in the SM Higgs sector [2].

Measurements of the fiducial cross sections and of several differential distributions using the $8 \mathrm{TeV}$ LHC data have been reported by CMS in Refs. [3, 4] for the $\mathrm{H} \rightarrow \mathrm{ZZ} \rightarrow 4 \ell(\ell=\mathrm{e}, \mu)$ and $\mathrm{H} \rightarrow \gamma \gamma$ decay channels. Here, we report a measurement of the integrated fiducial cross section times branching fraction $(\sigma \times \mathscr{B})$ and transverse momentum spectrum for the Higgs boson production using $\mathrm{H} \rightarrow \mathrm{W}^{+} \mathrm{W}^{-} \rightarrow \mathrm{e}^{ \pm} \mu^{\mp} v v$ decays, based on $\sqrt{s}=8 \mathrm{TeV}$ LHC data [5]. The $\mathrm{H} \rightarrow \mathrm{WW} \rightarrow 2 \ell 2 v$ channel is characterized by a lower resolution in the $p_{\mathrm{T}}^{\mathrm{H}}$ measurement compared to the $\mathrm{H} \rightarrow \gamma \gamma$ and $\mathrm{H} \rightarrow \mathrm{ZZ} \rightarrow 4 \ell$ channels because of neutrinos in the final state. However this channel has a significantly larger $\sigma \times \mathscr{B}$, exceeding that for the $\mathrm{H} \rightarrow \gamma \gamma$ by a factor of 10 and $\mathrm{H} \rightarrow \mathrm{ZZ} \rightarrow 4 \ell$ by a factor of 85 for a Higgs boson mass of $125 \mathrm{GeV}$, and is characterized by a good signal sensitivity.

The measurement is performed in a fiducial phase space that closely matches the experimental event selection in terms of lepton kinematics and event topology. The effect of the limited detector resolution, as well as the selection efficiency with respect to the fiducial phase space, are corrected to the particle level with an unfolding procedure.

The analysis presented here is based on the previously published $\mathrm{H} \rightarrow \mathrm{WW} \rightarrow 2 \ell 2 \mathrm{v}$ measurements by CMS [6]. A noticeable difference from those measurements is that this analysis is inclusive in the number of jets, which allows to reduce the uncertainties related to the theoretical modelling of additional jets produced in association with the Higgs boson.

\section{Data and simulated samples}

The analysis makes use of data recorded by the CMS experiment during 2012 that correspond to an integrated luminosity of $19.4 \mathrm{fb}^{-1}$ at a centre-of-mass energy of $8 \mathrm{TeV}$. The events used in this analysis are triggered by requiring the presence of either one or a combination of electron and muon with high $p_{\mathrm{T}}$ and tight identification and isolation criteria.

Simulated events of the Higgs boson production through the gluon fusion $(\mathrm{ggH})$ and vector boson fusion (VBF) mechanisms are performed using the POWHEG V1generator, which provides NLO accuracy calculation in perturbative QCD.

The main background processes, nonresonant $\mathrm{q} \overline{\mathrm{q}} \rightarrow \mathrm{W}^{+} \mathrm{W}^{-}$and $\mathrm{t} \overline{\mathrm{t}}+$ jets, are simulated using the MadGraph 5.1.3and Powheg V1 event generators respectively. The same Monte Carlo (MC) programs are used to simulate other minor background processes. All signal and background generators are interfaced to PYTHIA 6 to simulate the effects of the parton shower, multiple parton interactions, and hadronization. The samples are processed using a simulation of the CMS detector response, as modeled by GEANT4. 


\section{Analysis strategy}

The basic requirement for the analysis selection consists in two isolated leptons with opposite charge, an electron and a muon, with $p_{\mathrm{T}}$ greater than 20 (10) $\mathrm{GeV}$ for the leading (subleading) lepton, and with $|\eta|<2.5(|\eta|<2.4)$ for electrons (muons). No additional electron or muon with $p_{\mathrm{T}}>10 \mathrm{GeV}$ is allowed. The two leptons must originate from a single primary vertex and the invariant mass of the two leptons, $m_{\ell \ell}$, is required to be greater than $12 \mathrm{GeV}$. A projected $E_{\mathrm{T}}^{\text {miss }}$ variable is defined as the component of $\vec{p}_{\mathrm{T}}$ miss transverse to the nearest lepton if the lepton is situated within the azimuthal angular window of $\pm \pi / 2$ from the $\vec{p}_{\mathrm{T}}$ miss direction, or the $E_{\mathrm{T}}^{\text {miss }}$ itself otherwise [6]. Since the $E_{\mathrm{T}}^{\text {miss }}$ resolution is degraded by pileup, the minimum of two projected $E_{\mathrm{T}}^{\text {miss }}$ variables is used: one constructed from all identified particles (full projected $E_{\mathrm{T}}^{\text {miss }}$ ), and another constructed from the charged particles only (track projected $E_{\mathrm{T}}^{\text {miss }}$ ). Events must have both $E_{\mathrm{T}}^{\text {miss }}$ and the minimum projected $E_{\mathrm{T}}^{\text {miss }}$ above $20 \mathrm{GeV}$. The vector $p_{\mathrm{T}}$ sum of the two leptons, $p_{\mathrm{T}}^{\ell \ell}$, is required to be greater than $30 \mathrm{GeV}$ and a minimum transverse mass of the lepton plus $E_{\mathrm{T}}^{\text {miss }}$ vector of $60 \mathrm{GeV}$ is required. The transverse mass is defined as $\left.\left.m_{\mathrm{T}}=\sqrt{2 p_{\mathrm{T}}^{\ell \ell} E_{\mathrm{T}}^{\text {miss }}\left(1-\cos \Delta \phi\left(\ell \ell, \vec{p}_{\mathrm{T}} \text { miss }\right.\right.}\right)\right)$, where $\Delta \phi\left(\ell \ell, \vec{p}_{\mathrm{T}}\right.$ miss $)$ is the azimuthal angle between the dilepton momentum and $\vec{p}_{\mathrm{T}}$ miss.

The fiducial phase space is defined at the particle level and the requirements have been chosen to closely match the analysis event selection in order to minimize the dependence of the measurements on the underlying model of the Higgs boson properties and its production mechanism. The leptons are defined as Born-level leptons, i.e. before the emission of final state radiation (FSR), and are required to not come from leptonic $\tau$ decays. The requirements for the fiducial phase space definition are summarized in Table 1.

Table 1: Summary of requirements used in the definition of the fiducial phase space.

\begin{tabular}{lr}
\hline \multicolumn{1}{c}{ Kinematic requirements for the $\mathrm{H} \rightarrow \mathrm{WW} \rightarrow 2 \ell 2 v$ fiducial phase space } \\
\hline Leading lepton $p_{\mathrm{T}}$ & $p_{\mathrm{T}}>20 \mathrm{GeV}$ \\
Sub-leading lepton $p_{\mathrm{T}}$ & $p_{\mathrm{T}}>10 \mathrm{GeV}$ \\
Pseudorapidity of electrons and muons & $|\eta|<2.5$ \\
Invariant mass of the two leptons & $m_{\ell \ell}>12 \mathrm{GeV}$ \\
Transverse momentum of the lepton pair & $p_{\mathrm{T}}^{\ell \ell}>30 \mathrm{GeV}$ \\
Invariant mass of the leptonic system in the transverse plane & $m_{\mathrm{T}}>50 \mathrm{GeV}$ \\
No $E_{\mathrm{T}}^{\text {miss }}$ cut applied & \\
\hline
\end{tabular}

Events surviving these requirements are dominantly those where a top-antitop quark pair is produced and both quarks decay leptonically (dileptonic $\mathrm{t} \overline{\mathrm{t}}$ ). These events are classified using a combination of $\mathrm{b}$ tagging algorithms and rejected requiring to have no jets with $p_{\mathrm{T}}>30 \mathrm{GeV}$ identified by the algorithms. Events containing jets with $15<p_{\mathrm{T}}<30 \mathrm{GeV}$ are also rejected if containing a b-tagged jet. In this latter category a soft-muon veto is applied, rejecting the events containing a nonisolated low $p_{\mathrm{T}}$ muon, that is likely coming from a $\mathrm{b}$ hadron decay. The contribution of the dileptonic $\mathrm{t} \overline{\mathrm{t}}$ production is estimated independently in each $p_{\mathrm{T}}^{\mathrm{H}}$ bin, by measuring the $\bar{t} \bar{t}$ normalization in a control region enriched in $\mathrm{b}$ jets, obtained requiring at least two jets with $p_{\mathrm{T}}>30 \mathrm{GeV}$, one of which characterized by a large value of the $\mathrm{b}$ tagging discriminator. Another 
important background is nonresonant $W W \rightarrow 2 \ell 2 v$ production, which is also determined independently in each $p_{\mathrm{T}}^{\mathrm{H}}$ bin. The shape of this background in each bin is taken from the simulation, while its normalization is determined from data, owing to the different $\left(m_{\ell \ell}, m_{\mathrm{T}}\right)$ shape with respect to the signal contribution. Other minor backgrounds are estimated using the techniques described in Ref. [6].

\section{Systematic uncertainties}

Systematic uncertainties and are divided into three categories: uncertainties in background predictions, uncertainties in the experimental measurements and theoretical uncertainties.

Methods for the estimation of most of the systematic uncertainties are taken from the $\mathrm{H} \rightarrow$ $\mathrm{WW} \rightarrow 2 \ell 2 v$ published analysis [6]. One of the main differences is in the uncertainties related to the prediction of the contributions from $\mathrm{t} \overline{\mathrm{t}}$ and $\mathrm{tW}$ processes. The shapes of those backgrounds are corrected for different $b$ tagging efficiency in data and simulation and the normalization is taken from data in a top-quark enriched control region independently for each $p_{\mathrm{T}}^{\mathrm{H}}$ bin.

The analysis takes into account the theoretical uncertainties that affect the normalization and shape of all backgrounds predicted using simulations as well as the signal distribution shape. These are uncertainties from the missing higher-order corrections in perturbative QCD, and those related to the PDF uncertainties.

Since the $\left(m_{\ell \ell}, m_{\mathrm{T}}\right)$ shape of the WW background contribution is taken from simulation, the corresponding shape uncertainty is estimated comparing different theoretical calculations and including the effect of considering different QCD scales.

\section{Signal extraction and unfolding}

The signal, including ggH, VBF and $\mathrm{VH}$ production mechanisms, is extracted in each bin of $p_{\mathrm{T}}^{\mathrm{H}}$ performing a simultaneous binned maximum likelihood fit in the $m_{\ell \ell}-m_{\mathrm{T}}$ plane. The reconstructed spectrum is corrected for detector effects using an unfolding procedure that relies on the Singular Value Decomposition [7] method based on the Tikhonov regularization function. The response matrix used in the unfolding procedure is shown in Fig. 1(a). The treatment and the propagation of the systematic uncertainties through the unfolding procedure is performed differently depending on whether the source of uncertainty affects only the signal yield, both the signal yield and the response matrix, or only the response matrix. The latter are referred to as uncertainties in the signal model, and are evaluated using a different signal model for building a response matrix, e.g. varying the $\mathrm{VBF} / \mathrm{ggH}$ ratio with respect to the SM.

\section{Results}

The unfolded spectrum of $p_{\mathrm{T}}^{\mathrm{H}}$ is shown in Fig. 1(b). Statistical, systematic and theoretical uncertainties are shown as separate error bands in the plot. Data are in agreement with the theoretical predictions of HRES and POWHEG V2.

Due to the unfolding procedure and to the systematic uncertainties, the neighbouring bins in the spectrum have a sizeable level of correlation. 


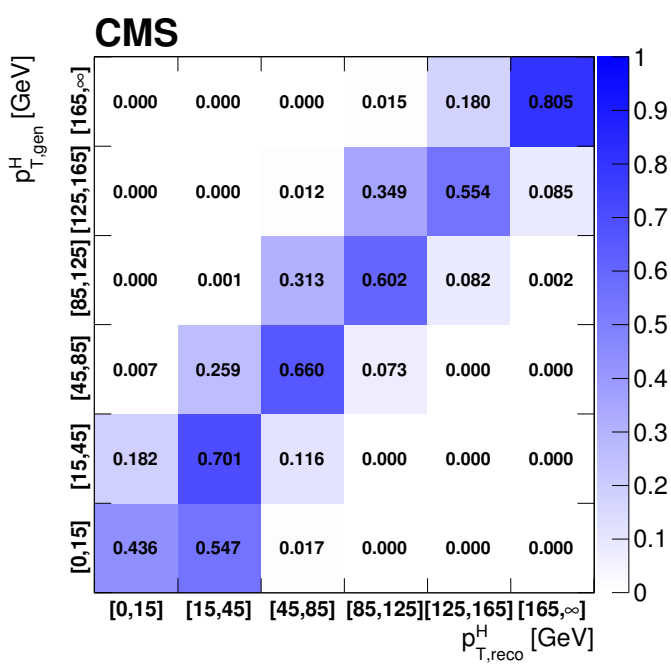

(a)

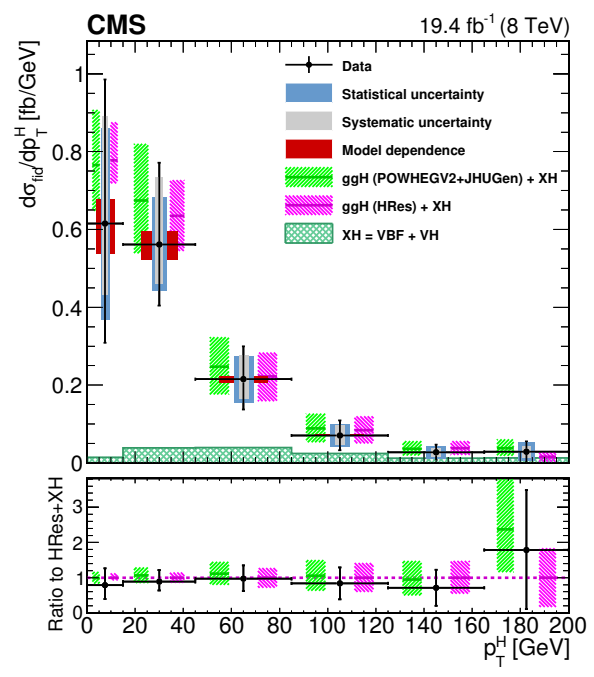

(b)

Figure 1: (a) Response matrix including all signal processes. The matrix is normalized by row in order to emphasize the resolution effects. (b) Unfolded Higgs boson production cross section as a function of $p_{\mathrm{T}}^{\mathrm{H}}$. Data points are shown, together with statistical and systematic uncertainties. The model dependence uncertainty is also shown. Data are compared to the ggH SM theoretical estimates from HRES and POWHEG $\mathrm{V} 2$. The sub-dominant component of the signal is denoted as $\mathrm{XH}=\mathrm{VBF}+\mathrm{VH}$ and it is also shown separately.

The inclusive fiducial cross section times branching fraction is measured to be:

$$
\sigma_{\text {fid }}=39 \pm 8 \text { (stat) } \pm 9 \text { (syst) fb , }
$$

in agreement within the uncertainties with the POWHEG V 2 theoretical estimate of $48 \pm 8 \mathrm{fb}$.

\section{References}

[1] R. V. Harlander and T. Neumann, Probing the nature of the Higgs-gluon coupling, Phys. Rev. D 88 (2013) 074015 [hep-th/1308.2225]

[2] M. Grazzini and H. Sargsyan, Heavy-quark mass effects in Higgs boson production at the LHC, JHEP 09 (2013) 129 [hep-ph/1306.4581]

[3] CMS Collaboration, Measurement of differential cross sections for Higgs boson production in the diphoton decay channel in pp collisions at $\sqrt{s}=8 \mathrm{TeV}$, Eur. Phys. J. C 76 (2013) 13 [hep-ex/1508.07819]

[4] CMS Collaboration, Measurement of differential and integrated fiducial cross sections for Higgs boson production in the four-lepton decay channel in pp collisions at $\sqrt{s}=7$ and 8 TeV, JHEP 04 (2016) 005 [hep-ex/1512.08377]

[5] CMS Collaboration, Measurement of the transverse momentum spectrum of the Higgs boson produced in pp collisions at $\sqrt{s}=8 \mathrm{TeV}$ using $H \rightarrow W W$ decays Submitted to JHEP (2016) [hep-ex/1606.01522]

[6] CMS Collaboration, Measurement of Higgs boson production and properties in the WW decay channel with leptonic final states, JHEP 01 (2014) 096 [hep-ex/1312.1129]

[7] A. Hocker and V. Kartvelishvili, SVD Approach to Data Unfolding, Nucl. Instrum. Meth. A 372 (1996) 469 [hep-ph/9509307] 